\begin{tabular}{lllllllll}
$\mathbf{A}$ & $\mathbf{R}$ & $\mathbf{T}$ & $\mathbf{Y}$ & $\mathbf{K}$ & $\mathbf{U}$ & $\mathbf{L}$ & $\mathbf{Y}$ \\
\hline
\end{tabular}

ROCZNIKI TEOLOGICZNE

Tom LXVII, zeszyt $6-2020$

DOI: http://dx.doi.org/10.18290/rt.20676-1

\title{
POUR UN PROCESSUS CATÉCHÉTIQUE SANS CESSE (NÉO-)CATÉCHUMÉNAL: UN ENGENDREMENT À TOUT ÂGE
}

\author{
FOR A CONTINUOUS CATECHETICAL PROCESS (NEO-)CATECHUMENAL: \\ A BEGETTING AT ANY AGE
}

\begin{abstract}
A bstract. How can we deal with the double dimension of Christian initiation, which is both ended when it is "completed" and open to the future of Christian existence? In our postmodern era of fluidity and blurring of the ages of life, catechetical pastoral care is invited to transform itself by offering multiple entry doors for the preparation of the sacraments, since we can be baptized and receive the sacrament of confirmation at any age. This transformation is accomplished by making each sacramental journey and the whole of existence an unceasingly (neo-)catechumenal journey where the first-second kerygmatic announcement is each time repeated and deepened. It is so, because, according to an eschatological aim, the possible moments of spiritual maturity are numerous in life and the pedagogy of permanent initiation leads to a maturation never fully accomplished.
\end{abstract}

Key words: initiation; catechumenate; ages; fluidity; life in the Spirit; maturity; maturation; joy of the Gospel; accompaniment; intergeneration; profession of faith; first announcement; second announcement; rites of passage; commitment; sacrament; baptism; confirmation; Eucharist.

François-Xavier Amherdt est prêtre du diocèse de Sion (Valais - Suisse). Ancien vice-directeur du séminaire et vicaire épiscopal de son diocèse, il a été curé-doyen de Sierre et Noës, puis directeur de l'Institut romand de Formation aux Ministères à Fribourg. Depuis 2007, il est professeur francophone de théologie pastorale, pédagogie religieuse et homilétique à l'Université de Fribourg (Suisse). Il est co-responsable du Comité italo-helvétique de la rédaction et directeur-adjoint de Lumen Vitae; e-mail : francois-xavier.amherdt@unifr.ch; ORCID: http://orcid.org/0000-0001-5488-8584

FrAnÇOIS-XAVIER AMHERDT - prof. dr hab., prezbiter diecezji Sion (Valais w Szwajcarii). Kierownik Specjalności Teologii Praktycznej, były wicerektor seminarium i wikariusz biskupi diecezji diecezji Sion. Był proboszczem i dziekanem Sierre i Noësa, a następnie dyrektorem Institut Romand de Formation aux Ministries we Fryburgu. Od 2007 roku jest francuskojęzycznym profesorem teologii pastoralnej, pedagogiki religijnej i homiletyki na uniwersytecie we Fryburgu (Szwajcaria). Współprzewodniczący włosko-szwajcarskiego komitetu redakcyjnego i zastępca dyrektora Lumen Vitae; e-mail: francois-xavier.amherdt@unifr.ch; ORCID: http://orcid.org/0000-0001-5488-8584 


\section{INTRODUCTION : LE DÉFI DE L'INITIATION CHRÉTIENNE}

\subsection{BALISES DE CROISSANCE}

Le défi de l'initiation chrétienne, à l'heure des mutations anthropologiques de notre société liquide, postmoderne et transhumaniste, consiste à réussir à tenir ensemble deux dimensions :

- d'une part, la nécessité de proposer certaines balises et étapes, certes plus flexibles que par le passé, mais pourtant indispensables pour permettre à chacun de structurer son récit de vie personnel et à l'Église d'organiser le vivre ensemble qui fait sa communion (clôture de l'initiation);

- d'autre part, la possibilité d'offrir une croissance spirituelle permanente tout au long de l'existence, vers un plus d'autonomie et de sagesse, en une conversion jamais achevée (ouverture de l'initiation).

C'est donc faire droit à la double perspective de clôture / ouverture de l'initiation, exactement comme le Canon des Écritures, dont la délimitation ecclésialement déterminée est source d'une créativité d'interprétations plurielles jamais tarie. Ainsi, l'initiation s'achève lorsque les trois sacrements sont reçus. Mais c'est à ce moment-là que tout commence, au point que la tradition spirituelle parle de « seconde conversion $»^{1}$, la recherche en théologie pastorale de $"$ seconde annonce $»^{2}$ et le Magistère de l'Église catholique de « nouvelle évangélisation $»^{3}$.

\subsection{FLUIDITÉ DES ÂGES DE L'EXISTENCE}

Notre thèse est que les apparentes perturbations des dispositions habituelles de l'initiation chrétienne, induites par le brouillage actuel des âges de l'existence, peuvent en fait constituer une chance pour la pratique ecclésiale de l'initiation sacramentelle. La fluidité des périodes de la vie amène en effet la pastorale catéchétique à mettre en place un cadre plus souple que par le passé, individuel et collectif, ponctué de seuils rituels, mais susceptible d'être abordé ou renouvelé à tout âge et dans les circonstances existentielles.

${ }^{1}$ François-Xavier AmHerdt, « La (seconde) conversion pastorale : une Pâque de l'action dans l'Esprit », dans : Guido Vergauven - Andreas Steingruber (Hgb.), Veni, Sancte Spiritus! Theologische Beiträge zur Sendung des Geistes - Contributions théologiques à la mission de l'Esprit - Theological Contributions to the Mission of the Spirit. Festschrift für Barbara Hallensleben zum 60. Geburtstag, Münster, Aschendorff, 2018, pp. 339-351.

${ }^{2}$ Enzo Biemmi, La seconde annonce. La grâce de recommencer, coll. « Pédagogie catéchétique », n. 29, Namur, Lumen Vitae, 2014.

${ }^{3}$ JeAn-Paul II, Novo Millennio ineunte (Au début du nouveau millénaire), Lettre apostolique au terme du grand Jubilé de l'an 2000, Rome, 2001. 
L'adoption par la Conférence des évêques de France de la pédagogie d'initiation, adaptée au rythme de chacun, mise en œuvre de manière personnalisée et déployée dans une catéchèse de cheminement communautaire pour toutes les générations ${ }^{4}$ (dans la Lettre aux catholiques de France de $1996^{5}$ et le Texte national pour l'orientation de la catéchèse en France de $2006^{6}$ ), renoue en fait avec les grandes options patristiques du catéchuménat antique. Cette orientation est partagée par la plupart des Conférences épiscopales d'Europe et son bien-fondé se voit officiellement reconnu dans le Directoire général pour la catéchèse de $1997^{7}$ et dans la récente exhortation du pape François Evangelii gaudium ${ }^{8}$. Le pontife argentin requiert en effet une catéchèse à la fois kérygmatique de première (seconde) annonce, aussi bien que mystagogique, c'est-à-dire articulée à la liturgie, portée par la communauté et parlant à toutes les dimensions de la personne (EG, n. 163-168).

\subsection{UNE PORTÉE ESCHATOLOGIQUE}

Une telle visée de proposition de la nouveauté de l'Évangile à tout âge, en tout contexte, et d'accompagnement individualisé respectant la progression de chacun.e, cherche à mettre en place les conditions de possibilité pour que chaque être puisse être petit à petit engendré à son identité humaine et spirituelle. Une telle pastorale initiatique d'engendrement se déploie en un véritable parcours de « développement personnel spirituel $»^{9}$. Ce processus constamment initiatique de croissance et de « fortification de l'homme intérieur » (Éphésiens 3,16), que l'évêque de Rome évoque également pour le couple et la famille (dans Amoris laetitia) ${ }^{10}$, dure bien toute l'existence.

${ }^{4}$ Luc Aerens, La catéchèse de cheminement. Pédagogie pastorale pour mener la transition en paroisse, coll. «Pédagogie catéchétique », n. 14, Namur, Lumen Vitae, 2003.

${ }^{5}$ ConfÉrence des ÉVÊQues de France, Proposer la foi dans la société actuelle. Lettre aux catholiques de France, Paris, Cerf, 1996.

${ }^{6}$ ConfÉrence des ÉvÊQues de France, Texte national pour l'orientation de la catéchèse en France et principes d'organisation, Paris, Bayard / Cerf / Fleurus-Mame, 2006 (cité TNOC). https:// catechese.catholique.fr/outils/conference-contribution/5547-2005-texte-national-lorientation-de-catechese-france-tnoc-principes-dorganisation/.

${ }^{7}$ CONGRÉGATION POUR Le Clergé, Directoire général pour la catéchèse, Paris / Bruxelles, Centurion / Cerf / Lumen Vitae, 1997 (cité DGC).

${ }^{8}$ Françors, Evangelii gaudium (La joie de l'Évangile), Exhortation apostolique sur l'annonce de l'Évangile dans le monde d'aujourd'hui, Rome, 2013 (cité $E G$ ).

${ }^{9} \mathrm{Cf}$. notre ouvrage avec Marie-Agnès De MatTeO, S'ouvrir à la fécondité de l'Esprit. Fondements d'une pastorale d'engendrement, coll. « Perspectives pastorales », n. 4, St-Maurice, Saint-Augustin, 2009.

${ }^{10}$ Françors, Amoris laetitia (La joie de l'amour), Exhortation apostolique post-synodale sur l'amour dans la famille, Rome, 2016. 
Il s'inscrit dans une perspective eschatologique de croissance et d' " écologie intégrale $»^{11}$, jusqu'à ce qu'advienne en chacun de nous "l'homme nouveau » (Éphésiens 4,21) et que tous, nous parvenions à former « cet homme parfait dans la force de l'âge qui réalise la plénitude du Christ » (Éphésiens 4,13).

Cette ouverture constante à la fécondité de l'Esprit concerne tout âge et ne s'achève jamais. Elle n'épuise pas la « réserve eschatologique » que contiennent les sacrements de l'initiation chrétienne. Les Écritures emploient diverses expressions pour la désigner : « redevenir comme un enfant », dans les synoptiques (Marc 9,$36 ; 10,16$ et parallèles) : « renaître de l'eau et de l'Esprit », dans le quatrième évangile (Jean 3,5); " grandir dans le langage raisonnable » (1 Corinthiens 13,11) et « devenir adulte dans le jugement» (1 Corinthiens 14,20), selon Paul.

$\mathrm{Au}$ fond, cette initiation à notre maturité spirituelle correspond, mutatis mutandis, à la « seconde naïveté postcritique » selon Paul Ricœur, c'est-à-dire à cette capacité d'émerveillement si profonde de l'enfant, redécouverte après avoir traversé pas à pas les déserts décapants de l'intelligence explicative. Selon l'adage cher au philosophe français : «Expliquer plus pour comprendre mieux $»^{12}$.

\subsection{STRUCTURE}

Au chapitre suivant (2), nous examinerons un certain nombre de brouillages survenus dans les pratiques ecclésiales d'initiation chrétienne, semblant bousculer les compartimentations claires qui semblaient exister auparavant. Puis nous dégagerons différentes ressources du massif de l'initiation chrétienne que recèle la Tradition patristique et actuelle, aptes à « rendre Dieu désirable » pour nos contemporains ${ }^{13}$, à répondre à leur soif spirituelle ${ }^{14}$, à leur permettre à chaque étape de recommencer ${ }^{15}$ et à faire de toute l'existence chrétienne un parcours sans cesse «néo-catéchuménal », jamais terminé (chap. 3).

${ }^{11}$ Cf. François, Laudato si' (Loué sois-tu), Lettre encyclique sur la sauvegarde de la maison commune, Rome, 2015.

${ }^{12} \mathrm{Cf}$. ma thèse L'herméneutique philosophique de Paul Ricœur et son importance pour l'exégèse biblique. En débat avec la New Yale Thelogy School, coll. « La nuit surveillée », Paris / St-Maurice, Cerf / Saint-Augustin, 2004.

${ }^{13}$ André Fossion, Dieu désirable. Proposition de la foi et initiation, coll. «Pédagogie catéchétique », n. 25, Namur / Montréal, Lumen Vitae / Novalis, 2010.

${ }^{14}$ François-Xavier AmHerdt (dir.), Pastorale et catéchèse : quelle spiritualité ?, Lumen Vitae 71, 1/2016, pp. 1-120.

${ }^{15}$ André Fossion, Une nouvelle fois. Vingt chemins pour recommencer à croire, Paris, L'Atelier, 2004. 


\section{PHÉNOMÈNES ECCLÉSIAUX DE BROUILLAGE DES ÉTAPES}

\subsection{UNE CATÉCHÈSE À TOUT ÂGE}

La mentalité selon laquelle « la catéchèse, c'est pour les enfants » n'a de loin pas disparu. Les parents sont encore souvent surpris quand les responsables ecclésiaux les sollicitent pour les prier d'assumer une partie de la catéchèse familiale ou paroissiale ${ }^{16}$. Ou plus encore, lorsqu'ils constatent qu'ils sont également visés par la catéchèse : «Tout ça, ce n'est pas pour nous. Nous avons déjà tout reçu ! 》 Or, affirme le $D G C$ (n. 59), la catéchèse d'adultes est la matrice du dispositif initiatique ecclésial, d'abord parce que c'est à l'âge adulte qu'il devient possible d'entrer en relation intime avec le Christ. Ensuite, parce que c'est dans la mesure où les parents se sentent eux-mêmes greffés sur le cep qu'est le Christ qu'ils peuvent entraîner leurs enfants à se laisser à leur tour habiter par la même sève de l'Esprit (cf. Jean 15,1-17). « On ne naît pas chrétien, on le devient », proclame l'adage de Tertullien. La catéchèse de cheminement familial et communautaire au fil de l'existence accompagne ce long travail initiatique de maturation ${ }^{17}$.

\subsection{BAPTISÉS ET CONFIRMÉS À TOUT ÂGE}

Autre source de surprise : on peut recevoir le baptême et/ou la confirmation à tout âge. Chaque année en France, près de 4’000 adultes sont initiés à la veillée pascale (quelques centaines en Suisse). Contrairement à ce que l'on véhicule dans les médias et l'opinion publique, ce n'est pas qu'à l'islam qu'on se convertit aujourd'hui, et en l'occurrence la préparation aux sacrements de l'initiation chrétienne n'a rien d'un chemin de radicalisation. Selon notre expérience, désormais presque dans un couple sur trois, l'un des fiancés n'est pas confirmé, et se voit donc invité à suivre un parcours explicitement catéchuménal avant ou après le mariage.

Les pratiques varient selon les diocèses et selon les régions de l'Europe et du monde. Certains évêques, comme celui de mon diocèse de Sion (en Suisse romande $)^{18}$, optent pour un rapatriement de la confirmation à l'âge de la maturité de l'enfance, entre 9 et 11 ans, dans une seule célébration groupée avec l'eucharistie.

\footnotetext{
${ }^{16}$ Gilles RouthiER, Sacrée catéchèse! Quand tu déranges familles et paroisses, coll. «Pédagogie catéchétique ", n 19, Bruxelles / Angers, Lumen Vitae / CRER, 2007.

${ }^{17}$ Henri Derroitte, Catéchèse et initiation, coll. «Pédagogie catéchétique », nº 18, Bruxelles, Lumen Vitae, 2005.

${ }^{18}$ Voir DiocèsE DE SiON, « Orientations diocésaines pour une pastorale catéchétique renouvelée. Vers une catéchèse d'ensemencement », http://www.cath-vs.ch/.
} 
D'autres au contraire continuent de distendre l'unité des trois sacrements de l'initiation chrétienne en proposant une première communion solennelle vers 9-10 ans, puis une profession de foi renouvelant celle du baptême vers 13-15 ans et une confirmation au début de l'âge adulte (16-18 ans) : ainsi en Suisse alémanique. D'autres évêques, comme celui de Lausanne, Genève et Fribourg laissent la possibilité de célébrer la confirmation entre 12 et 18 ans ${ }^{19}$, voire à tout âge, tout en offrant des démarches de type catéchuménal à dimension communautaire. «Ce flou entre les diocèses et les unités pastorales ne va-t-il pas troubler les gens ? », critiquent certains, et « déstabiliser notre pastorale, puisque nous avions pris l'habitude de célébrer pour tous la confirmation entre 16 et 18 ans ? », ajoutent des agents pastoraux.

À cette diversité entre régions et diocèses s'ajoute la pluralité des pratiques concernant les baptêmes d'adultes, certains diocèses tendant à privilégier ce que prévoit le Rituel de l'initiation chrétienne des adultes ${ }^{20}$, à savoir une célébration regroupant les trois sacrements, pour en souligner l'unité indissociable, d'autres préférant, pour des motifs pastoraux, laisser un espace de quelques semaines, par exemple le temps pascal, ou même d'une année, en célébrant une confirmation retardée ou décalée (notamment à la Pentecôte, si le baptême est fêté le Samedi saint), afin de pouvoir vivre un véritable temps de mystagogie et d'insérer réellement les néophytes dans la communauté où ils seront ensuite confirmés.

\subsection{DES ENGAGEMENTS PLUS TARDIFS}

Les demandes sacramentelles plus tardives, en réponse à la proposition large du baptême et de la confirmation ${ }^{21}$, correspondent au trend général de notre société selon lequel le mariage, s'il est encore sollicité, est envisagé aux alentours des trente ans, souvent quand vient le premier enfant. En outre, beaucoup de séminaristes, futurs diacres et religieux.ses se décident à entrer au séminaire, en formation ou au noviciat bien plus tard qu'autrefois. Avec d'ailleurs l'instauration d'une année de discernement dans les séminaires diocésains, ce qui révèle cette tendance très

\footnotetext{
${ }^{19}$ Voir Diocèse De Lausanne, Genève et Fribourg, « Orientations diocésaines en vue du cheminement vers la confirmation », https://www.diocese-lgf.ch/.

${ }^{20}$ ASSOCIATION ÉPISCOPALE LITURGIQUE POUR LES PAYS FRANCOPHONES, Rituel de l'initiation chrétienne des adultes (cité RICA), Paris, Desclée-Mame, 1996 et son Guide pastoral du Rituel de l'initiation chrétienne des adultes, édité par le SeRVICE NATIONAL DU CATÉCHUMÉNAT, coll. « Guides célébrer », Paris, Cerf, 2000.

${ }^{21}$ Commission épiscopale française de liturgie, Pastorale sacramentelle. Points de repères. Commentaires et guide de travail, T. I : Les sacrements de l'initiation chrétienne et le mariage, coll. « Liturgie », n 7, Paris, Cerf, 1996.
} 
répandue d'une adolescence - «adulescence » de plus en plus prolongée et d'une arrivée à la maturité adulte après un temps prolongé de «maturescence ». Qui de nos jours, dans notre société liquide ${ }^{22}$, dans notre univers hyper-connecté, où tout bouge et tout change sans cesse, peut se dire sorti de l'adulescence prolongée, parvenu à la maturité de la foi et prêt à prononcer une promesse pour la vie ?

\subsection{DE JEUNES PERSONNES ÂGÉES}

Phénomène corollaire, fruit du « jeunisme » (ou « syndrome de Peter Pan ») et du désir de rester toujours jeunes, beaux, musclés et bronzés, d'innombrables retraités paraissent remarquablement jeunes et peuvent constituer d'excellents piliers pour les adolescents, selon la bonne mode des « ânés dans la foi $»^{23}$ en tant que témoins de la foi pour les confirmants par exemple, bien souvent en substitution sur ce registre aux parrains et marraines qui demeurent assez « folkloriques ». Le brouillage est encore renforcé par l'augmentation quantitative exponentielle des quatrième et cinquième âges, avec le défi pour notre pastorale de donner un sens évangélique à ces étapes que la société regarde de plus en plus comme inutiles (aide au suicide, euthanasie, sélection de ceux qui ont le droit encore de vivre ou techniques transhumanistes en vue de rendre l'homme immortel ${ }^{24}$, etc.).

\section{DES TRANSFORMATIONS FRUCTUEUSES}

Comment dès lors répondre pastoralement à ces remises en cause d'un certain ordre établi ? Ma thèse est qu'en puisant au patrimoine biblique et patristique, la pastorale catéchétique peut transmuer ces variations troublantes au premier abord en perspectives fructueuses et bénéfiques. C'est par la fidélité à la Tradition ecclé-

${ }^{22}$ Voir à propos de la notion de « société liquide» les travaux de Zygmunt BAUmAN : La vie en miettes. Expérience postmoderne et moralité, Rodez, Le Rouergue / Chambon, 2003 ; L'amour liquide. De la fragilité des liens entre les hommes, Rodez, Le Rouergue / Chambon, 2004 ; Le présent liquide. Peurs sociales et obsession sécuritaire, Paris, Seuil, 2007 ; Identité, Paris, L'Herne, 2010 ; et pour l'Église, ceux d'Arnaud Jorn-Lambert : « Vers une Église «liquide» », Études 4213 (2/2015), pp. 67-78 ; «La mission chrétienne en modernité liquide. Une pluralité nécessaire », Études 4241 (9/2017), pp. 73-82.

${ }^{23}$ Cf. Conférence des ÉvêQues de France, Proposer la foi dans la société actuelle. Lettre aux catholiques de France et Texte national pour l'orientation de la catéchèse en France et principes d'organisation.

${ }^{24}$ Cf. Luc Ferry, La révolution transhumaniste, Paris, Plon, 2016 ; Thierry Magnin, Penser l'humain au temps de l'homme augmenté, Paris, Albin Michel, 2017. 
siale que la pratique de l'initiation chrétienne aujourd'hui peut s'avérer innovante, créatrice et pertinente. Énumérons quelques-unes de ces pistes.

\subsection{LA PÉDAGOGIE D’INITIATION}

Fondamentalement, je crois que la mise en œuvre de la pédagogie d'initiation héritée des Pères n'a de loin pas encore produit tous ses fruits, que ce soit en lien avec l'année liturgique, les milieux de vie, les étapes de l'existence et les demandes sacramentelles - selon les quatre types de contextes énumérés dans le $T N O C^{25}$.

\subsubsection{OUVERTURE DE L'INITIATION CHRÉTIENNE}

Premièrement, il s'agit de faire en sorte que l'unité de l'initiation soit mieux perçue, avec les deux premiers sacrements donnés seulement une fois, si possible conférés dans l'ordre traditionnel baptême - confirmation - eucharistie, et le troisième fait pour être répété et nourrir les deux autres.

Il convient ainsi de faire jouer la complémentarité entre baptême et confirmation, la seconde permettant de vivre la redditio de la profession de foi, du Notre Père et de la Parole de Dieu, en rendant ce qui a été reçu, en traditio, lors du premier. De plus, il est nécessaire de renforcer la valeur initiatique de l'eucharistie, en la plaçant en principe au troisième position, en atténuant la dimension d'unicité de ladite « première communion » et en valorisant sa signification de clôture / ouverture de tout l'édifice initiatique, en tant que « première des communions » nourrissant les baptisés-confirmés.

\subsubsection{DES RITES DE PASSAGES}

Bien des anthropologues déplorent la disparition dans nos sociétés occidentales de rites de passages facilitant la transition vers l'âge adulte et la traversée de l'existence. Or, les sacrements de l'initiation chrétienne peuvent être chargés d'une telle signification, puisqu'ils comportent, comme tout rite de ce type, une séparation des catéchumènes présentés comme tels à la communauté, un temps de mise à l'écart durant leur préparation-retraite et une nouvelle agrégation à l'assemblée avec leur statut de membres à part entière. Ces trois moments doivent aussi constituer de véritables étapes catéchuménales, soulignés en tant que telles.

\footnotetext{
${ }^{25}$ Dans sa seconde partie, « Principes d'organisation », pp. 37-54 (en ligne : voir note n. 6).
} 


\subsubsection{UN MODÈLE À RÉPÉTER}

Cœur de notre thèse : le catéchuménat des adultes (cf. le RICA) offre un modèle réduit du parcours de vie potentiel de tout être, considéré soit sur l'ensemble de sa trajectoire, soit pour chacune des tranches de son déploiement:

1) L'entrée en catéchuménat correspond à l'ouverture d'une période de réflexion et de croissance marquée par des avancées ou des hésitations. Elle peut durer plus ou moins longtemps, être ponctuée par différentes sortes de parcours ou des moments forts, des events, qui en relancent la ferveur. Comme les autres étapes, elle se vit individuellement et communautairement : chacun.e a son rythme personnel et tous ont besoin d'être soutenus par des groupes ou la communauté paroissiale.

2) Puis l'appel décisif, au début des quarante jours du Carême, indique un temps d'ultime préparation en vue d'un engagement. Cette période est scandée par la célébration des « scrutins », pour laisser Dieu scruter le cœur des candidats et les libérer de tout ce qui les empêche d'être encore eux-mêmes. Cette période cruciale, ponctuée par les traditiones et les redditiones du livre de la Parole, du Notre Père et du Credo ${ }^{26}$, correspond aux moments décisifs de l'existence, à l'adolescence, lors de choix de vie, aux carrefours personnels et professionnels, aux brisures et réconciliations, aux ruptures avec le mal et la violence. Ils peuvent faire l'objet de séquences ou rencontres plus spécifiques, parfois uniques, à partir desquelles tout le reste se joue.

3) Quant à la célébration des sacrements à la veillée pascale, elle est suivie de temps de mystagogies (telles celles de Cyrille de Jérusalem, Jean Chrysostome ou Ambroise de Milan) ${ }^{27}$, où les néophytes relisent les saints mystères en communauté, pénètrent plus avant dans leur intelligence et découvrent comment vivre dans l'Esprit au quotidien, dans la durée (Romains 8,1-17). À ce suivi mystagogique peuvent correspondre toutes les procédures (néo-)catéchuménales, dont nous reparlerons ${ }^{28}$, et qui courent le reste de l'existence jusqu'à la vieillesse.

\subsection{4. À L'OCCASION DES DIVERS MOMENTS DE MATURITÉ}

La pédagogie d'initiation s'ancre donc dans un certain nombre de points d'appuis $^{29}$ qui favorisent la croissance intérieure et la construction du Royaume. Aux différentes étapes, elle met en jeu des procédures :

\footnotetext{
${ }^{26}$ Cf. supra, 3.1.1, « Ouverture de l'initiation chrétienne ».

${ }^{27}$ Cf. Coll., L'initiation chrétienne hier et aujourd'hui, coll. " Connaissance des Pères de l’Église », n. 152, Bruyères-le-Châtel, Nouvelle Cité, 2018.

${ }^{28} \mathrm{Cf}$. infra, 3.1.5, « Un (néo-)catéchuménat permanent ».

${ }^{29}$ TNOC, chapitre 3, « Les points d'appui d'une pédagogie d'initiation en catéchèse », pp. 26-33 (en ligne : voir note n. 6).
} 
1) s'adressant à la liberté et à l'individu ;

2) touchant les multiples facettes de la personne, le corps et les sens, l'âme et le cœur, l'intelligence et l'esprit ;

3) par toute la richesse symbolique des langages de l'Écriture ;

4) et de la Tradition;

5) de la liturgie et de la communauté, ou des cheminements (néo-)catéchuménaux;

6) favorisant une décision et un choix libres ;

7) au sein d'une société multiculturelle et pluri-religieuse.

Elle s'applique donc à tous les âges, puisque la Parole, la Tradition, les sacrements, la foi, la prière, le mystère pascal, n'ont pas d'âge.

Par conséquent, il est légitime de proposer le dispositif initiatique aux différents moments de maturité de l'être humain : au sommet « métaphysique » de l'enfance entre 8 et 12 ans, avant les mutations physiologiques de la puberté ; au cœur des transformations de la première adolescence, pour accompagner les transitions pascales qui s'y expérimentent ; dans le frémissement de la jeunesse, lorsque se posent quelques-unes des orientations professionnelles, personnelles ou affectives importantes pour la suite ; quand viennent les maturations attachées aux responsabilités économiques, sociétales, maritales ou familiales ; aux temps des remises en cause du « démon de midi » et des changements de caps ; lorsque se vit une seconde conversion spirituelle, où à la tendance $d u$ « faire des choses pour Dieu » succède un véritable abandon à la volonté du Seigneur, un « se laisser faire par Dieu » ${ }^{30}$; lors d'une prise de conscience, d'une rencontre-clé, d'un événement décisif qui suscite un « recommencement dans la foi $»^{31}$.

\subsubsection{UN (NÉO-)CATÉCHUMÉNAT PERMANENT}

Dans cette perspective, nous pourrions dire que la pastorale et la vie chrétienne tout entière se résument à un « (néo-)catéchuménat permanent » qui n’a jamais fini de déployer ses potentialités, selon la dynamique eschatologique du « déjà / pas encore ». La catéchèse se conçoit de cette manière comme une constante réactuali-

${ }^{30} \mathrm{Cf}$. notre ouvrage édité avec Roland LACROIX - Équipe Européenne de Catéchèse, La conversion : l'acte, le processus, l'accompagnement. Actes du Congrès de l'Équipe Européenne de Catéchèse, Celje (Slovénie), 27 mai - 1er juin 2015, coll. «Perspectives pastorales », n. 12, St-Maurice, Saint-Augustin, 2019.

${ }^{31}$ Voir toute la littérature qui a fleuri ces dernières années autour des « recommençants », entre autres : H. BourgeoIs, À l'appel des recommençants. Évaluations et propositions, coll. «Vivre, Croire, Célébrer », Paris, L'Atelier, 2001 ; Redécouvrir la foi. Les recommençants, coll. " Pascal Thomas - Pratiques chrétiennes », Paris, DDB, 1993 ; R. LACRoIX, Revisiter la foi chrétienne avec les recommençants, Paris, L'Atelier, 2002. 
sation du mystère pascal ${ }^{32}$, dont la grâce est entièrement donnée au baptême-confirmation, et n'a de cesse de se manifester tout au long de l'existence, par le pain de l'eucharistie, de la Parole, de la prière et du service.

Cette extrême souplesse, cette fluidité pastorale théologiquement motivée, munit les agents pastoraux d'outils diversifiés, appropriés à toutes les circonstances et étapes de l'existence. Ainsi, l'initiation peut se faire, se renouveler, se poursuivre à tout moment, parce que nous sommes initiés par les sacrements et le trésor ecclésial, et non seulement $\grave{a}$ eux.

À cet égard, il est d'une importance décisive de prévoir pour tous les parcours sacramentels un temps de suivi mystagogique, faisant dès le début partie intégrante de la démarche. Les célébrations sacramentelles viennent ainsi « en cours de route » et ne sont plus conçues comme le terme de la procédure, telles des récompenses « méritées » grâce à l'obtention d'un certain nombre de « point-crédits » spirituels (cf. $E G$, n. 40). L'initiation chrétienne, c'est comme le permis de conduire ou le mariage - et l'ordination : c'est après que cela commence !

\subsection{UNE INITIATION AU SERVICE DE LA MATURITÉ}

\subsubsection{LES SACREMENTS : VERS QUELLE MATURITÉ ?}

De ce fait, ne faudrait-il pas renverser la question, en se demandant non plus quelle maturité est requise pour « obtenir» un sacrement, mais à quelle maturité les étapes proposées conduisent-elles, sur le chemin de l'existence ?

Il est significatif à cet égard de constater que l'Église canonise des saint(e)s de tout âge, y compris des enfants et des jeunes ${ }^{33}$ ! Quelle conversion il faut encore accomplir pour que la pastorale catéchétique prenne vraiment ce visage faisant de chaque préparation (mariage, baptême des enfants, onction, funérailles) l'occasion d'un « (néo-)catéchuménat post-baptismal et eucharistique » ${ }^{34}$.

\subsubsection{DES PROFESSIONS DE FOI}

Nous pensons qu'il serait par exemple opportun de multiplier les célébrations de la profession de foi lors d'une série de carrefours de l'existence (début de l'apprentissage

\footnotetext{
${ }^{32}$ Cf. notre essai Le mystère pascal. Aller au cour de la foi, Bière, Cabédita, 2019.

${ }^{33}$ Voir Françors, Christus vivit, Exhortation apostolique post-synodale aux jeunes et à tout le peuple de Dieu, Rome, 2019, n. 49-63, « Des jeunes saints ».

${ }^{34}$ Ainsi que le préconise le Décret sur l'activité missionnaire du concile Vatican II Ad Gentes, n. 14, qui parle d'un « catéchuménat post-baptismal » nécessaire à ceux qui ont été baptisés bébés.
} 
et première paye, fiançailles et mariage, venue du premier enfant, obtention d'un diplôme, changement de métier, même rupture de couple et remariage civil, etc.) et d'offrir de nouveaux rites anthropologiques " néo-baptismaux » à forte densité théologico-symbolique pour soutenir les passages existentiels ${ }^{35}$.

\subsubsection{L'ANNÉE LITURGIQUE}

La pédagogie de l'année liturgique offre à ce point de vue un modèle de répétition sans cesse renouvelé, comme un ressort allant toujours plus haut ou plus profond.

\subsection{LA PREMIÈRE-SECONDE ANNONCE}

Tout ce qui est de l'ordre de festivals, récollections, temps forts (comme lors des Journées Mondiales de la Jeunesse, internationales, nationales ou régionales) devrait ainsi se concevoir dans la visée d'une première ou seconde (première) annonce ${ }^{36}, c^{\prime}$ est-à-dire de la possibilité de se laisser toucher à nouveau par la grâce originelle vivace du mystère pascal. Les expériences humaines fondamentales en sont des occasions privilégiées : enfanter et laisser partir, chercher et se tromper, se passionner et être en relation, entrer en compassion, souffrir et affronter la maladie et la mort ${ }^{37}$.

\subsection{LA CATÉCHÈSE INTERGÉNÉRATIONNELLE}

L'Église est depuis toujours un réseau, un web avant l'heure. Toutes les démarches initiatiques sont confiées à la responsabilité de l'ensemble des communautés, appelées à être à la fois catéchisées et catéchisantes ${ }^{38}$, avec tous, par tous et pour tous. C'est mutuellement que les diverses générations peuvent s'enrichir, la fraîcheur des uns stimulant la sagesse des autres. D’où ces journées intergénération-

\footnotetext{
${ }^{35}$ Comme s'y emploient de manière paradigmatique Henri Derroitte et Maurice Queloz avec leur ouvrage Langage symbolique et catéchèse communautaire, coll. «Pédagogie catéchétique », n. 22, Bruxelles, Lumen Vitae, 2008.

${ }^{36}$ Enzo Biemmi - André Fossion, La conversion missionnaire de la catéchèse. Proposition de la foi et première annonce, coll. « Pédagogie catéchétique », n² 24, Bruxelles, Lumen Vitae, 2009.

${ }^{37} \mathrm{Cf}$. ma chronique, « Le projet de seconde annonce : un laboratoire des diocèses italiens », Lumen Vitae 73, 3/2018, pp. 349-354.

${ }^{38}$ Bill Huebsch, La catéchèse de toute la communauté. Vers une catéchèse par tous, avec tous et pour tous, coll. «Pédagogie catéchétique », n 17, Bruxelles / Paris / Montréal, Lumen Vitae / Bayard / Novalis, 2001.
} 
nelles sur l'Esprit, le pardon, l'eucharistie, etc., où toute la communauté est conviée, avec la messe au centre, des ateliers par tranches d'âges, des débats et échanges tous ensemble, des temps de repas ${ }^{39}$.

\subsection{ACCOMPAGNEMENT PERSONNALISÉ}

La flexibilité des parcours initiatiques implique de multiplier les portes d'entrée possibles, pour les catéchumènes de différents âges et types, les recommençants, les chercheurs de sens. Elle implique une pluralité de parcours et de formules qui exige aussi une démultiplication des agents pastoraux. Elle requiert un véritable accompagnement personnalisé, se rattachant à la tradition de l'accompagnement spirituel ${ }^{40}$. Les Exercices spirituels de saint Ignace de Loyola en sont l'un des modèles les plus achevés. Ils peuvent se vivre à n'importe quel moment de l'existence, avant une décision vitale, après un certain nombre d'années d'activité, après une rupture pour un nouveau départ.

L'initiation chrétienne se conjugue donc volontiers aussi avec la pratique de l'écriture de son récit de vie : cette relecture, partielle ou globale, consiste en un essai de symbolisation, au sens fort de sun-ballô, mettre ensemble les morceaux épars, et de configuration de l'identité narrative ${ }^{41}$. Un tel exercice permet de conférer une cohésion rétroactive à une tranche de vie ou à la globalité de l'existence, de soigner certaines blessures par l'expérience du pardon, et de conduire parfois à une renaissance, à un engendrement par la grâce à sa véritable vocation ${ }^{42}$.

\footnotetext{
${ }^{39}$ Voir mes essais : « La catéchèse intergénérationnelle et communautaire - entre les générations et au sein de la communauté » [Catechesis across generations and within community], Bildungsforschung 1 (2017), https://open-journals4.uni-tuebingen.de/ojs/index.php/bildungsforschung/issue/ view/25 ; «Dans la ligne d'Amoris laetitia : le modèle des journées de catéchèses intergénérationnelles », dans François-Xavier AmHERDT - Roland LACRoIX (éds.), Équipe Européenne de Catéchèse, La famille : entre éducation et proposition de la foi. Actes du Congrès de l'Équipe Européenne de Catéchèse, Madrid, 31 mai - 5 juin 2017, coll. " Perspectives pastorales », n. 13, St-Maurice, Saint-Augustin, 2020.

${ }^{40}$ Cf. François-Xavier Амнerdt (dir.), Vers une culture du discernement, Lumen Vitae 75, 1/2020, pp. 1-120.

${ }^{41}$ Selon la terminologie chère à Paul Ricœur des trois mimèsis : préfiguration, configuration et refiguration.

${ }^{42} \mathrm{Cf}$. François-Xavier Amherdt, «Une œuvre de maturité », Préface à : Dieudonné Mushipu Мвомво, Le récit du pèlerin de saint Ignace de Loyola et son rôle formatif dans l'accompagnement spirituel, coll. « Théologie pratique en dialogue », n. 41, Fribourg, Academic Press, 2014, pp. 11-16.
} 


\section{DANS LA JOIE}

Au fond, considérée sous cet angle de vue, l'initiation chrétienne se présente comme une conversion jamais achevée, qui tend à la maturation de la liberté intérieure par le dépouillement progressif de l'homme ancien. Elle conduit à la « joie de l'Évangile » que nul ne peut nous ravir (Jean 16,22).

\section{BIBLIOGRAPHIE}

Aerens Luc, La catéchèse de cheminement. Pédagogie pastorale pour mener la transition en paroisse, coll. « Pédagogie catéchétique », n. 14, Namur, Lumen Vitae, 2003.

AMHERDt François-Xavier (dir.), Vers une culture du discernement, Lumen Vitae 75, 1/2020, pp. 1-120.

AMHERDT François-Xavier, « Dans la ligne d'Amoris laetitia : le modèle des journées de catéchèses intergénérationnelles », dans François-Xavier AMHERDT - Roland LACroIx (éds.), Équipe Européenne de Catéchèse, La famille : entre éducation et proposition de la foi. Actes du Congrès de l'Équipe Européenne de Catéchèse, Madrid, 31 mai - 5 juin 2017, coll. «Perspectives pastorales », n. 13, St-Maurice, Saint-Augustin, 2020.

AMHERDt François-Xavier, Le mystère pascal. Aller au cœur de la foi, Bière, Cabédita, 2019.

AMHERDT François-Xavier - Lacroix Roland (éds.), Équipe Européenne de Catéchèse, La conversion : l'acte, le processus, l'accompagnement. Actes du Congrès de l'Équipe Européenne de Catéchèse, Celje (Slovénie), 27 mai - $1^{\text {er }}$ juin 2015, coll. "Perspectives pastorales », n. 12, St-Maurice, Saint-Augustin, 2019.

AmHeRDt François-Xavier, « La (seconde) conversion pastorale : une Pâque de l'action dans l'Esprit », dans : Guido Vergauven - Andreas Steingruber (Hgb.), Veni, Sancte Spiritus ! Theologische Beiträge zur Sendung des Geistes - Contributions théologiques à la mission de l'Esprit - Theological Contributions to the Mission of the Spirit. Festschrift für Barbara Hallensleben zum 60. Geburtstag, Münster, Aschendorff, 2018, pp. 339-351.

AMHERDT François-Xavier, «Le projet de seconde annonce : un laboratoire des diocèses italiens », Lumen Vitae 73, 3/2018, pp. 349-354.

AMHERDT François-Xavier, « La catéchèse intergénérationnelle et communautaire - entre les générations et au sein de la communauté » [Catechesis across generations and within community], Bildungsforschung 1 (2017), https://open-journals4.uni-tuebingen.de/ojs/index.php/bildungsforschung/issue/view/25 (3.02.2020).

AMHERDT François-Xavier (dir), Pastorale et catéchèse : quelle spiritualité ?, Lumen Vitae 71, 1/2016, pp. 1-120.

Amherdt François-Xavier, Une œuvre de maturité, Préface à : Dieudonné Mushipu Mbombo, Le récit du pèlerin de saint Ignace de Loyola et son rôle formatif dans l'accompagnement spirituel, coll. «Théologie pratique en dialogue », n. 41, Fribourg, Academic Press, 2014, pp. 11-16.

AmHerdt François-Xavier - De Matteo Marie-Agnès, S'ouvrir à la fécondité de l'Esprit. Fondements d'une pastorale d'engendrement, coll. «Perspectives pastorales », n. 4, St-Maurice, Saint-Augustin, 2009.

AmHeRDt François-Xavier, L'herméneutique philosophique de Paul Ricœur et son importance pour l'exégèse biblique. En débat avec la New Yale Thelogy School, coll. « La nuit surveillée », Paris / St-Maurice, Cerf / Saint-Augustin, 2004.

ASSOCIATION ÉPISCOPALE LITURGIQUE POUR LES PAYS FRANCOPHONES, Guide pastoral du Rituel de l'initiation chrétienne des adultes, édité par le Service national du catéchuménat, coll. «Guides célébrer », Paris, Cerf, 2000. 
ASSOCIATION ÉPISCOPALE LITURGIQUE POUR LES PAYS FRANCOPHONES, Rituel de l'initiation chrétienne des adultes, Paris, Desclée-Mame, 1996.

Bauman Zygmunt, Identité, Paris, L'Herne, 2010.

Bauman Zygmunt, Le présent liquide. Peurs sociales et obsession sécuritaire, Paris, Seuil, 2007.

Bauman Zygmunt, L'amour liquide. De la fragilité des liens entre les hommes, Rodez, Le Rouergue / Chambon, 2004.

Bauman Zygmunt, La vie en miettes. Expérience postmoderne et moralité, Rodez, Le Rouergue / Chambon, 2003.

Biemmi Enzo, La seconde annonce. La grâce de recommencer, coll. « Pédagogie catéchétique », n. 29, Namur, Lumen Vitae, 2014.

Biemmi Enzo - Fossion André, La conversion missionnaire de la catéchèse. Proposition de la foi et première annonce, coll. «Pédagogie catéchétique », n. 24, Bruxelles, Lumen Vitae, 2009.

Bourgeois Henri, À l'appel des recommençants. Évaluations et propositions, coll. « Vivre, Croire, Célébrer », Paris, L'Atelier, 2001.

Bourgeois Henri, Redécouvrir la foi. Les recommençants, coll. « Pascal Thomas - Pratiques chrétiennes », Paris, DDB, 1993.

Collectif, L'initiation chrétienne hier et aujourd'hui, coll. « Connaissance des Pères de l'Église », n. 152, Bruyères-le-Châtel, Nouvelle Cité, 2018.

Commission éPISCOPALE FRANÇAISE De liturgie, Pastorale sacramentelle. Points de repères. Commentaires et guide de travail, T. I : Les sacrements de l'initiation chrétienne et le mariage, coll. « Liturgie », n. 7, Paris, Cerf, 1996.

CONFÉRENCE DES ÉVÊQUeS DE FrAnCE, Texte national pour l'orientation de la catéchèse en France et principes d'organisation, Paris, Bayard / Cerf / Fleurus-Mame, 2006, https://catechese. catholique.fr/outils/conference-contribution/5547-2005-texte-national-lorientation-de-catechese-france-tnoc-principes-dorganisation/ (3.02.2020).

Conférence des ÉVÊQues de France, Proposer la foi dans la société actuelle. Lettre aux catholiques de France, Paris, Cerf, 1996.

CONGRÉGATION POUR Le Clergé, Directoire général pour la catéchèse, Paris / Bruxelles, Centurion / Cerf / Lumen Vitae, 1997.

Derroitte Henri - Queloz Maurice, Langage symbolique et catéchèse communautaire, coll. « Pédagogie catéchétique », n. 22, Bruxelles, Lumen Vitae, 2008.

Derroitte Henri, Catéchèse et initiation, coll. «Pédagogie catéchétique », n. 18, Bruxelles, Lumen Vitae, 2005.

Diocèse de Lausanne, Genève et Fribourg, « Orientations diocésaines en vue du cheminement vers la confirmation », https://www.diocese-lgf.ch/ (3.02.2020).

DiocèSE DE SiON, " Orientations diocésaines pour une pastorale catéchétique renouvelée. Vers une catéchèse d'ensemencement », http://www.cath-vs.ch/ (3.02.2020).

FERry Luc, La révolution transhumaniste, Paris, Plon, 2016.

Fossion André, Dieu désirable. Proposition de la foi et initiation, coll. « Pédagogie catéchétique », n. 25, Namur / Montréal, Lumen Vitae / Novalis, 2010.

Fossion André, Une nouvelle fois. Vingt chemins pour recommencer à croire, Paris, L'Atelier, 2004.

FrançoIs, Exhortation apostolique «Christus vivit », Rome, 2019.

FrançoIs, Exhortation apostolique « Amoris laetitia », Rome, 2016.

FrançoIs, Lettre encyclique « Laudato si” », Rome, 2015.

FrançOIs, Exhortation apostolique « Evangelii gaudium », Rome, 2013.

HuEBSCH Bill, La catéchèse de toute la communauté. Vers une catéchèse par tous, avec tous et pour tous, coll. « Pédagogie catéchétique », n. 17, Bruxelles / Paris / Montréal, Lumen Vitae / Bayard / Novalis, 2001.

Jean-Paul II, Lettre apostolique « Novo Millennio ineunte », Rome, 2001. 
JOIN-LAMBERT Arnaud, « La mission chrétienne en modernité liquide. Une pluralité nécessaire », Études 4241, 9/2017, pp. 73-82.

JoIN-LAMBERT Arnaud, Vers une Église «liquide», Études 4213, 2/2015, pp. 67-78.

LACroix Roland, Revisiter la foi chrétienne avec les recommençants, Paris, L'Atelier, 2002.

Magnin Thierry, Penser l'humain au temps de l'homme augmenté, Paris, Albin Michel, 2017.

RouthiER Gilles, Sacrée catéchèse! Quand tu déranges familles et paroisses, coll. « Pédagogie catéchétique », n. 19, Bruxelles / Angers, Lumen Vitae / CRER, 2007.

VATICAN II, Décret sur l'activité missionnaire de l’Église «Ad Gentes », Rome, 1965.

\title{
W KIERUNKU CIĄGŁEGO PROCESU KATECHETYCZNEGO (NEO-)KATECHUMENALNEGO: PŁODNOŚĆ W KAŻDYM OKRESIE ROZWOJOWYM
}

\author{
Streszczenie
}

Jak poradzić sobie z podwójnym wymiarem inicjacji chrześcijańskiej, która jest zarówno zamknięta, kiedy jest formalnie ,ukończona”, jak i otwarta na przyszłość chrześcijańskiej egzystencji? W postmodernistycznej erze płynności i mieszania się okresów rozwojowych życia człowieka katechetyczna opieka duszpasterska powinna ulec transformacji, oferując wiele nowych sposobów do przygotowania się na przyjęcie sakramentów, ponieważ można być ochrzczonym i otrzymać sakrament bierzmowania w każdym okresie życia. Transformacja ta dokonuje się wówczas, gdy każda sakramentalna droga i całe życie staną się nieustanną drogą (neo-)katechumenalną, w której pierwsze i wtórne głoszenie kerygmatu jest za każdym razem wznawiane i pogłębiane. Dzieje się tak, ponieważ zgodnie z celem eschatologicznym możliwe momenty duchowej dojrzałości są liczne w życiu, a pedagogika ciągłego wtajemniczania prowadzi do dojrzewania, którego nigdy nie osiągnie się w pełni.

Słowa kluczowe: inicjacja; katechumenat; wiek; płynność; życie w Duchu; dojrzałość; dojrzewanie; radość Ewangelii; towarzyszenie; międzypokoleniowość; wyznanie wiary; pierwsza ewangelizacja; druga ewangelizacja; obrzędy przejścia; zaangażowanie; sakrament; chrzest; bierzmowanie; Eucharystia.

\section{POUR UN PROCESSUS CATÉCHÉTIQUE SANS CESSE (NÉO-)CATÉCHUMÉNAL: UN ENGENDREMENT À TOUT ÂGE}

\section{Résumé}

Comment faire droit à la double dimension de l'initiation chrétienne à la fois close lorsqu'elle est « achevée » et ouverte sur l'avenir de l'existence chrétienne ? Dans notre époque postmoderne de fluidité et de brouillage des âges de la vie, la pastorale catéchétique est invitée à se transformer en offrant de multiples portes d'entrée pour la préparation aux sacrements, puisqu'on peut être baptisé et confirmé à tout âge ; en faisant de chaque parcours sacramentel et de l'existence tout entière un cheminement sans cesse (néo-)catéchuménal où la première-seconde annonce kérygmatique est à chaque fois reprise et approfondie. Car selon une visée eschatologique, les moments possibles de maturité spirituelle sont nombreux dans une vie et la pédagogie d'initiation permanente conduit à une maturation jamais pleinement accomplie.

Mots-clés: Initiation; catéchuménat; âges; fluidité; vie dans l'Esprit; maturité; maturation; joie de l'Évangile; accompagnement; intergénération; profession de foi; première annonce; seconde annonce; rites de passages; engagement; sacrement; baptême; confirmation; Eucharistie. 